\title{
A technical note: When Do Differences in IMRT Fluence Intensities have Clinical Significance?
}

\author{
Yousif Y AM* and AJ Van Rensburg \\ Department of Radiation Oncology, Steve Biko Academic Hospital, South Africa
}

Submission: February 08, 2018; Published: March 27, 2018

"Correspondence Address: Yousif Y AM, Department of Radiation Oncology, Steve Biko Academic Hospital, Private Bage X169, Pretoria, 0001, South Africa, Email: Yousif.medphy@gmail.com

Abstract

The purpose of this exercise was to test the validity of gamma pass and fail criteria in order to develop a better method for clinical evaluation. This was performed through introducing artificial changes into a clinical IMRT plan and then followed by recalculation and evaluation. Although, the results showed that the IMRT plan has failed to meet the gamma evaluation criteria, the plan was still clinically acceptable when assessed by clinicians. This proved that the passing rate for IMRT fluence maps QA occasionally may not associated with the clinical significance of the plan.

Keywords: IMRT; Gamma criteria; Fluence map; QA

\section{Introduction}

The intensity modulated radiation therapy (IMRT) has been the treatment modality for several cancers' treatment sites [1,2]. The beauty of the technique, its ability to maximize the dose to the target volume while further reduces the dose to the organ at risk. Although the technique provides a good treatment outcome but it quality assurance has of the challenges in the verifications of the dose delivery [3,4]. At Steve Biko Academic Hospital the IMRT was implemented for the treatment of the head and neck cancer which is the most prevalent among the male in South Africa. The quality assurance of the technique in terms of the verification of the dose delivery has been the most concern. This is usually done through comparing the calculated IMRT plan using the planning system and the measured on with different dosimetric modalities such as film dosimetry and 2D-Array detector.

The satisfactory criteria are usually achieved through comparing the dose difference and the distance to agreement point by point [5]. This criterion is known a gamma index method in which most of the dose difference and distance to agreement are defaulted as $3 \%$ and $3 \mathrm{~mm}$ respectively [6]. The IMRT plan will be satisfactory even if as little as $90 \%$ of the tested points comply with the gamma criterion [6,7]. When comparing the dose distributions using the Gamma evolution criteria; the reasoning for using a $3 \% / 3 \mathrm{~mm}$ cut-off and the need that a certain percentage must pass e.g $90 \%$ is based rather on what is technically achievable and not what would have a clinical impact. The aim of this exercise was to see if a plan that fails the fluence QA with the current criteria and $90 \%$ would it be clinically unacceptable.

\section{Methods}

A three field head-and-neck IMRT plan generated using CMS XiO TPS was used (Elekta, Ltd, UK). The plan was chosen from the clinically approved and treated IMRT plans. The plan has multiple target volumes and multiple organs at risk that are distributed throughout the treatment volume. This plan was considered to be an error-free IMRT plan and was used for the IMRT QA dose plans and patient doses. Another IMRT plan was created by introducing errors into the chosen plan. The errors were introduced into the plan to create dose differences in the IMRT fluence maps. Artificial changes of more than $10 \%$ of the fluence area in the field were made by a value of $+5 \%$ in areas of a high dose gradient and dose levels in the field to simulate a few mis-calibrated leaves; these changes were followed by a recalculation of the IMRT plan. For fluence QA purposes, IMRT plans were recalculated in a homogenous water-density phantom. A 2D-Array Seven 29 ionization chamber (PTW, Freiburg, Germany) detector system with was used for IMRT $\mathrm{QA}$ verifications. The error-free plan and the error-induced plan were compared in terms of fluence maps and with the dosevolume histograms (DVHs). Finally, the suitability of both IMRT plans in terms of their dose matrices and patient anatomy dose differences were assessed; the clinicians in our department assisted with the assessments.

\section{Results and Discussion}

Figures 1 \& 2 show comparisons between the 2D-Array and TPS dose distributions and the gamma evaluation for the errorfree plan and the error-induced plan. These results enabled the 


\section{Cancer Therapy \& Oncology International Journal}

evaluation of the degree of agreement between the measured and calculated dose distributions in the regions of the dose gradient. Level-based assessment criteria were used for dose distribution comparisons. The criteria would only allow a 1st pass; 1 st pass and 2nd pass only; or $1^{\text {st }}, 2^{\text {nd }}$ and $3^{\text {rd }}$ pass. Details of $1^{\text {st }}, 2^{\text {nd }}$ and $3^{\text {rd }}$ pass definitions are provided by Depuydt et al. [7]. By using level-based assessment, the acceptance criteria were user definable with user inputs for distance-to-agreement (in $\mathrm{mm}$ ) and percentage dose difference. The dose range to which the distance-to-agreement and percentage difference matching criteria are applied vary (i.e., the user can elect to use a higher percentage tolerance below a nominated threshold of the total dose).

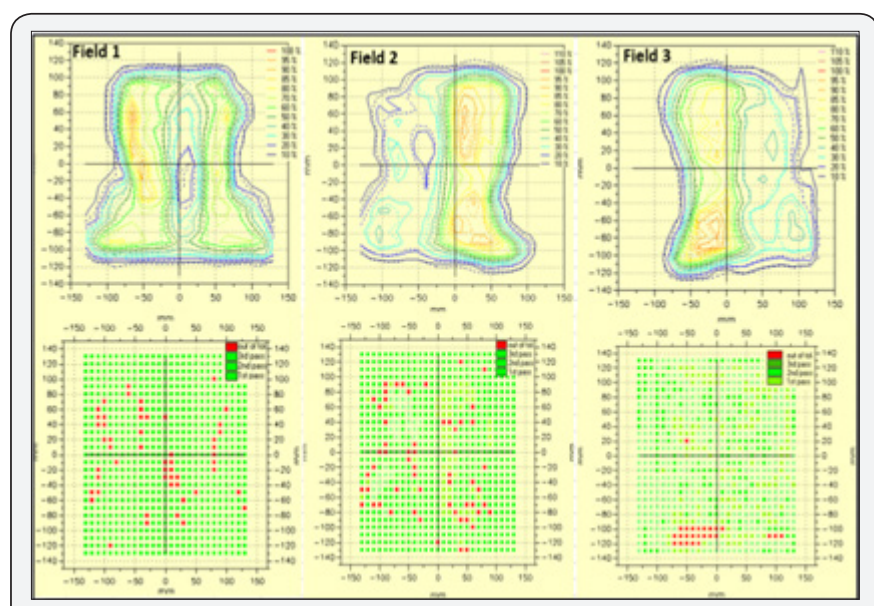

Figure 1: The isodose overlay (solid line: TPS; dotted lines: 2D-array) and gamma function pass/fail criteria for (a) the errorfree plan IMRT plan.

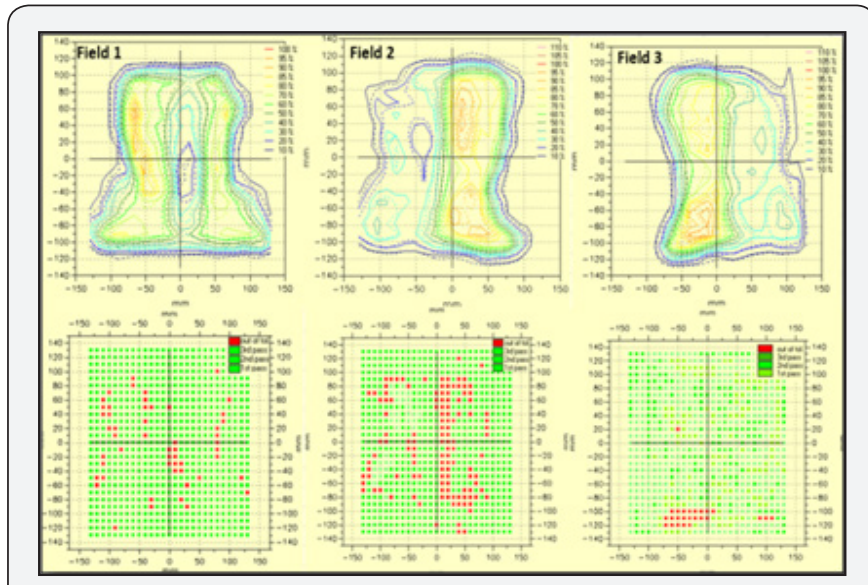

Figure 2 : The isodose overlay (solid line: TPS; dotted lines: $2 \mathrm{D}$-array) and gamma function pass/fail criteria for (b) the errorinduced IMRT plan.

The currently used version of the VeriSoft gamma evaluation does not report any numerical solutions of the gamma function; instead, a pass/fail criterion was implemented. The agreements were quantitatively confirmed by the gamma evaluation criteria, in which the green indicates the area or point that met the criteria, whereas the red indicates the points at which the criteria were not reached. Figure 1 depicts an error-free IMRT plan; the gamma evaluation plot of the dose distributions shows an agreement for most of the points among the datasets for the three fields with the exception of small deviations shown at the edges of the field. The disagreements resulted from the high dose gradient. Figure 2 depicts an IMRT plan with introduced errors. Field 1 and field 3 passed by most of the points. Field 2 exhibits the most of the points in the field that did not meet the evaluation criteria. These results showed that the IMRT plan failed to meet the criteria of $3 \% / 3 \mathrm{~mm}$.

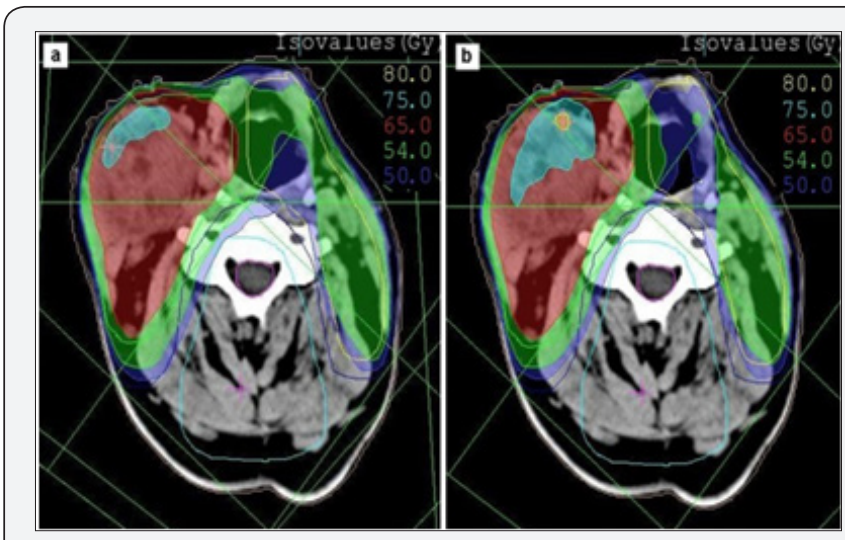

Figure 3 : The isodose distributions in an axial view in a patient with head and neck cancer.

a. The error-free IMRT plan;

b. The IMRT plan with errors.

The colors wash represent sets at $80,75,65,54$ and $50 \mathrm{~Gy}$.

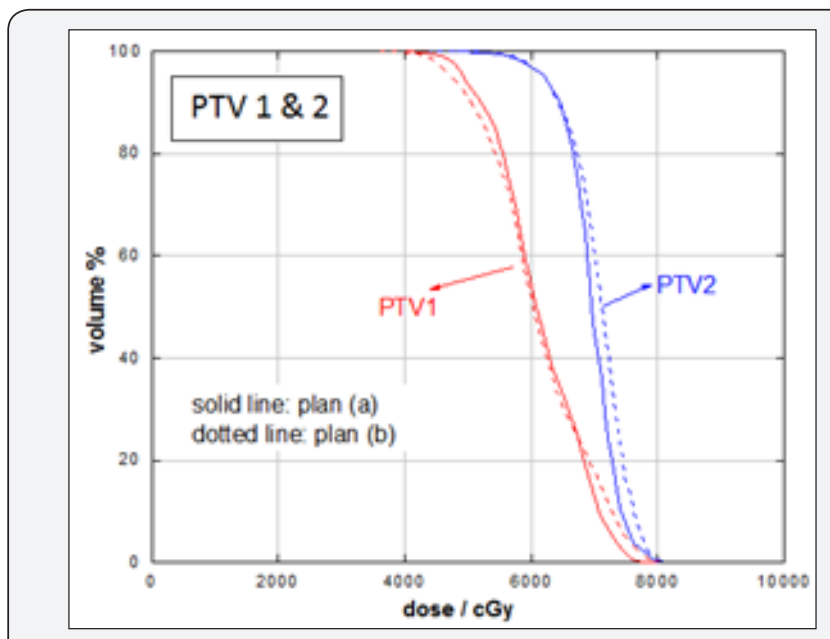

Figure 4 : A dose-volume histogram of differences between (a) the error-free IMRT plan (solid line) and (b) the IMRT plan with errors (dashed line). The DVH shows PTV1 and PTV2.

Figure 3 shows representative axial CT slides that indicate the isodose distributions that were obtained for the two IMRT plans. Both of the IMRT plans met the prescription goal for PTV1 and PTV2. For error-free plan (a), the deviations of $D_{90}$ (Gy) from the prescribed dose were 2.38 and 0.98 Gy for PTV1 and PTV2, respectively. For error-induced plan (b), the deviations of $\mathrm{D}_{90}$ (Gy) from the prescribed dose were 3.38 and 0.90 Gy for PTV1 and PTV2, respectively. The mean and the maximum dose differences between PTV1 and PTV2 were less than 2\%. Tables 1 \& 2 lists the $\mathrm{D}_{100}, \mathrm{D}_{90}, \mathrm{~V}_{100}, \mathrm{~V}_{90}$, mean and maximum dose 
values for target volumes for both plans. Table 2 summarizes the maximum dose received by the organs-at-risk, in which the OAR received doses lower the tolerance doses for each. The DVHs of the target volumes and of the organs-at-risk for both plans are shown in Figures 4 \& 5, respectively. The results demonstrated that both IMRT plans were acceptable.

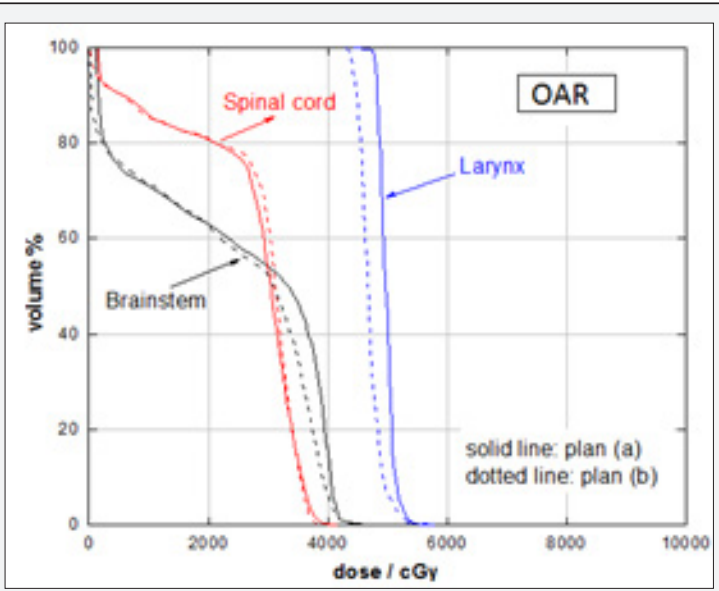

Figure 5 : A dose volume histogram of differences between the (a) error-free IMRT plan (solid line) and the (b) IMRT plan with errors (dashed line). The DVH shows the organs at risk.

Table 1: A dosimetric summary of the target volumes for plan (a) and (b).

\begin{tabular}{|c|c|c|c|c|}
\hline Plan & \multicolumn{2}{|c|}{ a } & \multicolumn{2}{c|}{ b } \\
\hline Parameters & PTV1 & PTV2 & PTV1 & PTV2 \\
\hline D100 (Gy) & 22.15 & 38 & 21.35 & 36.76 \\
\hline D90 (Gy) & 51.62 & 64.02 & 50.62 & 64.1 \\
\hline V100 (\%) & 84.76 & 87.2 & 81.12 & 87.82 \\
\hline V95 (\%) & 91.34 & 95.58 & 87.94 & 94.7 \\
\hline Dmean (Gy) & 61.27 & 69.31 & 60.95 & 70.56 \\
\hline Dmax (Gy) & 82.72 & 80.72 & 81.16 & 81.16 \\
\hline
\end{tabular}

Table 2: A dosimetric summary of organs at risk for both plans.

\begin{tabular}{|c|c|c|}
\hline Plan & $\mathbf{a}\left(\mathbf{D}_{\max }(\mathbf{G y})\right)$ & $\mathbf{b}\left(\mathbf{D}_{\mathbf{m a}} \mathbf{x}(\mathbf{G y})\right)$ \\
\hline Brainstem & 47.38 & 46.61 \\
\hline Spinal cord & 41.17 & 39.62 \\
\hline Larynx & 57.76 & 57.35 \\
\hline
\end{tabular}

Overall, the evaluation criteria for IMRT plans were set to investigate if he passing rate fails would the plan still be clinically suitable. The error-free IMRT plan passed the set gamma criteria as expected. For the error-induced IMRT plan, the acceptance criteria were not met, and this were clearly noted in areas where errors were introduced into the chosen plan in field 2. Although the IMRT plan has failed the fluence QA, when, this plan was assessed by the clinician, looking at the DVHs of the target volumes and of the organs-at-risk for both plans, the outcomes showed that the both of the error-free and error-induced IMRT plan were acceptable. The obtained results agreed with the published literature $[8,9]$. Despite the fact that the IMRT plan fails the gamma passing, the plan is still clinically acceptable. This result indicated that there is a relatively weak correlation between the passing rate for the IMRT plan and the clinical relevance. In contrast, the conventional IMRT fluence QA method not sensitive in predicting IMRT QA errors $[8,9]$.

\section{Conclusion}

The main aim of this study was to test the validity of the criteria pass/fail and develop a better method for clinical evaluation. This was done by introducing and artificial changes in clinical plan, and these changes followed by recalculation and evaluation. From the results, based on the evaluation of IMRT clinical plans using the pass-rate criteria, the generally accepted norm for the use of a fluence map with pass/fail criteria does not have a sound foundation in clinical practice. A better approach would be to substitute the measured fluence map into the TPS and recalculate. If differences are not clinically significant, then continue with treatment; otherwise, redo the IMRT plan and QA.

\section{Conflict of Interest}

The authors have no conflict of interest to declare.

\section{Acknowledgement}

The authors would like to thank the staff at the Department Radiation Oncology, Steve Biko Academic Hospital for their help and cooperation.

\section{References}

1. Palta, S Kim, J Li, C Liu (2003) Intensity-Modulated Radiation Therapy: The State of Art. In: Palta JR, TR Mackie (Eds.), Medical Physics. Madison, Wisconsin, USA, pp. 593-612.

2. Ezzell GA, Galvin JM, Low D, Palta JR, Rosen I, et al. (2003) AAPM REPORT: Guidance document on delivery, treatment planning, and clinical implementation of IMRT: Report of the IMRT subcommittee of the AAPM radiation therapy committee. Med Phys 30(8): 2089-2115.

3. Ezzell GA, Burmeister JW, Dogan N, Lo Sasso TJ, Mechalakos JG, et al. (2009) IMRT commissioning: multiple institution planning and dosimetry comparisons, a report from AAPM Task Group 119. Med Phys 36(11): 5359-5373.

4. McKenzie EM, Balter PA, Stingo FC, Jones J, Followill DS, et al. (2014) Toward optimizing patient-specific IMRT QA techniques in the accurate detection of dosimetrically acceptable and unacceptable patient plans. Med Phys 41(12): 121702.

5. Low DA, Harms WB, Mutic S, Purdy JA (1998) A technique for the quantitative evaluation of dose distributions. Med Phys 25(5): 656661.

6. Low DA, Dempesy FJ (2003) Evaluation of the gamma dose distribution comparison method, Med Phys 30(9): 2455-2464.

7. Depuydt T, Van Esch A, Huyskens D (2002) A quantitative evaluation of IMRT dose distributions: refinement and clinical assessment of the gamma evaluation. Radiother Oncol 62(3): 309-319.

8. Nelms BE, Zhen H, Tome WA (2011) Per-beam, planar IMRT QA passing rates do not predict clinically relevant patient dose errors. Med Phys 38(2): 1037-1044.

9. Kruse JJ (2010) On the insensitivity of single field planar dosimetry to IMRT inaccuracies. Med Phys 37(6): 2516-2524. 
Your next submission with Juniper Publishers will reach you the below assets

- Quality Editorial service

- Swift Peer Review

- Reprints availability

- E-prints Service

- Manuscript Podcast for convenient understanding

- Global attainment for your research

- Manuscript accessibility in different formats ( Pdf, E-pub, Full Text, Audio)

- Unceasing customer service

Track the below URL for one-step submission https://juniperpublishers.com/online-submission.php 